\title{
Rechazo y victimización al alumnado con necesidad de apoyo educativo en primero de primaria
}

\author{
M $^{a}$ Inés Monjas*, Luis J. Martín-Antón ${ }^{1}$, Francisco-Juan García-Bacete ${ }^{2}$ y Ma Luisa Sanchiz ${ }^{2}$ \\ ${ }^{1}$ Universidad de Valladolid \\ ${ }^{2}$ Universitat de Castelló
}

Resumen: Este estudio pretende analizar el rechazo y victimización social que experimenta el alumnado con necesidad de apoyo educativo en comparación con sus compañeros. Participaron 1351 alumnos de primero de educación primaria de los que 253 (el 18.7\%) fueron etiquetados por sus profesores como alumnos con necesidad de apoyo educativo ya que presentaban dificultades que hicieron necesaria ayuda educativa especial y adicional a resto de compañeros. La información ha sido proporcionada por los iguales (tipología sociométrica, reputación social y calificación sociométrica), el profesorado (competencia social) y el propio alumno (victimización y competencia percibida). Los resultados indican que los alumnos con necesidad de apoyo educativo son más rechazados, tienen peor reputación social (más agresivos, más aislados y menos prosociales) y sus profesores les califican como menos competentes socialmente. Informan de que son victimizados con más frecuencia que sus compañeros y se autoperciben como menos competentes cognitivamente y menos aceptados por sus iguales. Todo ello revela una evidente caracterización de este alumnado como rechazado y excluido. Es más, los aspectos que definen el perfil del rechazo se ven intensificados cuando se trata de estos alumnos, motivos por los que se concluye con la necesidad de prestar especial atención y cuidado a este colectivo en riesgo.

Palabras clave: Rechazo entre iguales; necesidad de apoyo educativo; victimización; competencia percibida; reputación social; competencia social.

\section{Introducción}

Ser aceptado y querido por los compañeros, tener amigos y estar integrado en los diversos escenarios escolares, familiares, virtuales y de ocio, donde los niños y adolescentes viven y se relacionan, son tareas evolutivas básicas que se tienen que ir alcanzando para el logro de un óptimo desarrollo emocional, cognitivo y social. En este largo y complejo proceso la mayoría logran relaciones positivas y satisfactorias, pero algunos no participan o son apartados de esas interacciones y tienen experiencias negativas; entre ellos están los que son activa y sistemáticamente rechazados, excluidos y victimizados por sus iguales, situaciones que conllevan consecuencias muy perjudiciales para el desarrollo socioemocional y cognitivo (Bierman, 2004; Gifford-Smith y Brownell, 2003).

En las últimas décadas la pujante investigación sobre las relaciones entre iguales ha aumentado considerablemente el conocimiento sobre la naturaleza e importancia de fenómenos como el rechazo y el acoso entre iguales.

En cuanto al rechazo, los estudios realizados evidencian que implica sentimientos negativos (ignorancia, antipatía, desagrado...) del grupo hacia alguno de sus miembros que, por diversos motivos, no gusta a los demás. Esto supone

* Dirección para correspondencia [Correspondence address]: $\mathrm{M}^{\mathrm{a}}$ Inés Monjas Casares. Área de Psicología Evolutiva y de la Educación. Departamento de Psicología. Universidad de Valladolid. Po Belén s/n. 47011 Valladolid (España). E-mail: imonjas@psi.uva.es
Title: Rejection and victimization among first graders primary school with education support needs.

Abstract: This study aims to analyze the social rejection and victimization experienced by students with educational support needs compared to their peers. Participants were 1351 first graders primary school, 253 of them $(18.7 \%)$ were labeled by their teachers as students with educational support needs since they had difficulties which required special and additional educational support. The information has been provided by peers (sociometric typology, social reputation and sociometric rating), teachers (social competence) and the students themselves (victimization and perceived competence). The results indicate that students with educational support needs are more rejected, have worse social reputation (more aggressive, more isolated and less prosocial) and their teachers consider they are less competent socially. These students report that they are victimized more often than their peers and they perceive themselves as less competent cognitively and less accepted by their peers. This shows a clear characterization of these students as rejected and excluded. Moreover, the aspects that define the profile of rejection are amplified when applied to these students, which highlights the necessity of paying special and careful attention to this risk group.

Key words: Peer rejection; educational support needs; victimization; perceived competence; social reputation; social competence.

que el niño rechazado es apartado y excluido de las dinámicas entre iguales y pierde oportunidades del contacto y del aprendizaje social que tiene lugar con los compañeros, a la vez que consolida su mala reputación en el grupo entrando así en una espiral negativa. Todo ello confirma la implicación interpersonal y grupal y no sólo de las características individuales del niño rechazado (Coie, 1990; Escobar, FernándezBaena, Miranda, Trianes y Cowie, 2011; García Bacete, Sureda y Monjas, 2010). A este respecto se ha investigado mucho sobre los correlatos del tipo sociométrico rechazado habiéndose señalado cuatro patrones conductuales asociados; en primer lugar, bajas tasas de sociabilidad y de conducta prosocial; en segundo lugar, alta agresión y conducta disruptiva; en tercer lugar inmadurez y falta de atención y finalmente, ansiedad social y conductas de evitación (Bierman, 2004). Se sabe también que, entre un 10 y un $15 \%$ del alumnado es rechazado por sus compañeros (García Bacete, Sureda y Monjas, 2008) y que este estatus presenta cierta estabilidad (Cillessen, Bukowski y Haselager, 2000; Jiang y Cillessen, 2005).

$\mathrm{El}$ acoso entre iguales o bullying es conceptualizado como un tipo de violencia interpersonal que consiste en el maltrato y hostigamiento intencional y sistemático de un chico hacia otro que está indefenso y que se convierte en víctima, de forma que se produce una perversión de las relaciones entre iguales que dejan de ser paritarias y simétricas y se convierten en desequilibradas y reguladas por el esquema dominiosumisión entre intimidador y víctima (Avilés y Monjas, 2008; 
Cerezo, 2009; Cerezo y Ato, 2010; Garaigordobil y Oñederra, 2010; Monjas y Avilés, 2006).

Estos dos tópicos, que están teniendo actualmente un marcado papel en la investigación sobre desarrollo social, generalmente se han estudiado por separado ya que sus manifestaciones en cierto modo son distintas. No obstante hay autores como Juvonen y Gross (2004) que incluyen el bullying dentro de la más amplia categoría de rechazo, mientras que otros como Harris (2009) consideran que el rechazo social es un tipo de acoso emocional. Existe además cierta controversia respecto a si el rechazo contribuye a la victimización o viceversa (Lucas, Pulido y Solbes, 2011). Sintetizando el estado de la cuestión puede considerarse que son fenómenos no equivalentes, pero conceptual y empíricamente relacionados. Los estudios señalan una correlación de alrededor de .50. (Gifford-Smith y Brownell, 2003) y en ambos casos se trata de experiencias aversivas repetidas y muy dolorosas que privan al niño de las oportunidades de interacción con los iguales. Esto le afecta negativamente porque restringe sus prácticas sociales y le lleva a la exclusión social, lo que supone una seria amenaza para el bienestar emocional y relevantes consecuencias a corto, medio y largo plazo (Buelga, Cava y Musitu, 2012; Juvonen y Graham, 2001; Sánchez, Ortega y Menesini, 2012; Warden y Mackinnon, 2003).

\section{Alumnado con necesidad de apoyo educativo}

Para el presente estudio consideramos Alumnado con Necesidad de Apoyo Educativo (en adelante NAE) a aquellos alumnos que, durante el curso escolar, han recibido apoyo, refuerzo o ayuda educativa especial, adicional o distinta al resto de compañeros. Este apoyo, individual, o en grupos de dos o tres personas, ha sido proporcionado, fuera o dentro del aula de referencia, bien por el profesorado habitual del aula (tutor de su grupo u otros maestros de Educación Física, Matemáticas, Lengua o Inglés) o bien por profesorado especializado (Pedagogía terapéutica, Audición y lenguaje o Educación compensatoria). Son los alumnos que, a juicio del tutor y del equipo de profesorado del grupo, tienen una discrepancia respecto a sus compañeros y presentan dificultades, o riesgo de presentarlas, en diversos aspectos (cognitivos e intelectuales, conductuales, emocionales y sociales, físicos o sensoriales, de comunicación y lenguaje, de lectoescritura y rendimiento escolar). Es preciso subrayar que constituyen un grupo heterogéneo y de gran diversidad de características y necesidades (ORDEN EDU/1152/2010) y solo en contados casos, como es esperable en el primer curso de educación primaria, tienen diagnóstico de alumnado con necesidades educativas especiales o con discapacidad.

La revisión bibliográfica llevada a cabo pone de manifiesto que, pese a la dilatada investigación en torno al rechazo y al bullying, no se ha prestado suficiente atención al alumnado con NAE, grupo que por sus especiales características y circunstancias (debilidad, situación de desventaja, encontrarse en inferioridad de número, carencia de red de apoyo social...) tiene, de partida, una cierta vulnerabilidad y constitu- ye un colectivo en riesgo de sufrir el rechazo, el acoso y la exclusión en los grupos de iguales. Especialmente en nuestro país este tema no ha sido suficientemente investigado por lo que tendremos en cuenta las contribuciones hechas por autores de otros países, dejando constancia de la dificultad que entraña comparar investigaciones realizadas con distintos sistemas educativos, con diversos enfoques en las políticas inclusivas y hasta con ciertas diferencias conceptuales y terminológicas (Ruijs y Peetsma, 2009). Fundamentalmente, aunque no exclusivamente, se han analizado los estudios hechos en educación primaria, especialmente con los más pequeños, en contextos escolares ordinarios donde están incluidos los alumnos con alguna necesidad educativa especial, priorizando las investigaciones que utilizan técnicas sociométricas para la identificación del alumnado rechazado.

\section{Aceptación social del alumnado con necesidad de apoyo educativo}

Las investigaciones ponen de manifiesto, consistentemente, que altas proporciones de alumnado con NAE en aulas ordinarias son menos aceptados y más rechazados que sus iguales sin necesidades especiales (Baydik y Bakkaloglu, 2009; Estell et al., 2008; Frederikson, 2010; Frederikson, Simmonds, Evans y Soulsby, 2007; Nakken y Pijl, 2002: Nowicki, 2003; Pijl, 2007; Sabeh y Monjas, 2002, entre otros). Esta situación de rechazo y bajo estatus social se produce tanto para situación de juego como académica y se aprecia incluso en las situaciones en que son parte de la clase y tienen amigos (Frederickson y Furnham, 2004).

Se informa asimismo de que tienen más dificultades sociales (Nowicki, 2003), presentan más conducta solitaria y mayores tasas de aislamiento social (Kavale y Forness, 1996; Kemp y Carter, 2002), participan menos frecuentemente como miembros de subgrupos (Frostad y Pijl, 2007), tienen menos amigos y pueden experimentar mayor soledad que sus iguales (Pijl, Frostad y Flem, 2010; Whitney, Nabuzoka y Smith, 1992). Un aspecto bastante reiterado es que tienen déficits en habilidades sociales (Al-Yagon y Mikulincer, 2004; Estell et al., 2008; Kavale y Forness, 1996). Se señala también que tienen problemas de autoconcepto (Bakker y Bosman, 2003; Ruijs y Peetsma, 2009). Con alumnado con dificultades de aprendizaje Pijl y Frostad (2010) encontraron relación entre la baja aceptación y el bajo autoconcepto y Bakker, Denessen, Bosman, Krijger y Bouts (2007) descubren que los alumnos con dificultades de aprendizaje generales tenían auto-imagen más baja que los alumnos con dificultades de aprendizaje específicas, especialmente entre las chicas y para los alumnos integrados en centros ordinarios. Aunque la bibliografía es muy escasa a este respecto ya que se cuestiona la capacidad de autopercepción e información de estos alumnos.

Es conveniente dejar constancia de que estos hallazgos son similares en diferentes sistemas escolares y en distintos países como Inglaterra, España o Noruega, país con más experiencias y tradición en inclusión. También hay que reseñar 
que los resultados se repiten con distintas limitaciones como son discapacidad intelectual (Scheepstra, Nakken y Piel, 1999), deficiencia auditiva (Cambra y Silvestre, 2003), dificultades de aprendizaje (Bakker et al., 2007), deficiencia visual (Eguren, Gutiérrez, Herrero y López, 2006), autismo (Symes y Humphrey, 2010) y problemas de conducta (Mand, 2007). Es preocupante además que la evidencia sugiere que el rechazo es bastante estable a lo largo del tiempo (Estell et al., 2008).

En una dirección distinta hay otros estudios que presentan una imagen más positiva del alumnado con NAE en la red social de la clase. Algunos resultados desvelan que su posición social no difiere en gran medida de la de sus iguales (Koster, Pijl, van Houten y Nakken, 2007), aunque sí permanecen menos tiempo interactuando con sus compañeros y más tiempo en conducta solitaria y aislada (Kemp y Carter, 2002). Avramidis (2010) precisa que tienen relaciones de amistad y son miembros de subgrupos. Vaughn, Elbaum y Schumm (1996) encontraron que a principios de curso eran rechazados, pero a medida que avanzó el curso incrementaron su aceptación social y aumentaron el número de amistades reciprocas. Los resultados de Estell et al. (2008) son similares a los anteriores, aunque señalan que tienen un estatus más bajo respecto al número de nominaciones como mejor amigo y su popularidad es ligeramente más baja. Determinado trabajos sugieren que en ocasiones se juntan en grupos de alumnos semejantes a ellos, que generalmente también tienen déficits sociales, conductas agresivas y/o comportamiento antisocial (Farmer, 2000). Es preciso mencionar la postura de Frederikson (2010) que afirma que el alumnado con necesidades especiales puede ser tratado más favorablemente por sus compañeros y apunta que para los alumnos que tienen necesidades más evidentes, sus compañeros sin necesidades especiales aplican estándares más indulgentes y esperan menos beneficios y toleran más costes en la relación (Frederikson y Furnham, 2004).

Por último es importante indicar que en varios de los estudios aludidos se señala que la visión que los padres y los profesores tienen respecto a la posición social de los alumnos con NAE es más positiva que la que manifiestan y expresan los compañeros del grupo. Los adultos subestiman los problemas de interacción de estos alumnos, con lo cual no desarrollan estrategias de prevención o intervención. La idea de los profesores de apoyo, aunque es también positiva, se acerca más a la de los iguales (Koster et al., 2007; Pijl y Frostad, 2010).

\section{Bullying y alumnado con necesidad de apoyo edu- cativo}

Por lo que se refiere al bullying, y teniendo en cuenta que es limitada la investigación con esta población, los estudios evidencian que los alumnos con NAE tienen mayor riesgo y sufren altos niveles de acoso e intimidación de sus compañeros sin NAE (Bourke y Burgman, 2010; Frederikson, 2010; Heinricks, 2003; Nabuzoka y Smith, 1993; Rose, Monda-
Amaya y Espelage, 2011). Está situación ocurre en diversas discapacidades y necesidades especiales como evidencia Carter y Spencer (2006) que revisan once estudios que dividen en dos categorías de dificultades: las visibles (espina bífida, parálisis cerebral, disfemia, etc.) e invisibles (dificultades de aprendizaje, trastorno por déficit de atención con hiperactividad, problemas de conducta, etc.) y concluyen que todos los alumnos con necesidades especiales, visibles e invisibles, experimentan más acoso que sus iguales. Así se pone de manifiesto en deficiencia auditiva (Dixon, Smith y Jenks, 2004), dificultades de lenguaje (Hugh-Jones y Smith, 1999; Lindsay, Dockrell y Mackie y 2008), problemas motóricos (Lindsay y McPherson, 2010), dificultades de aprendizaje (Norwick y Nelly, 2004), síndrome de asperger (Granizo, Na y del Barrio, 2008; Hernández y van der Meulen, 2010), problemas de lenguaje (Evans, Healey, Hawai y Rowland, 2008; Savage, 2005) y discapacidad intelectual (Flynt y Morton, 2004). La única excepción se refiere a los alumnos ciegos totales que son menos acosados puntualizando que, en estos casos, la discapacidad es más un factor de protección que de riesgo (Eguren et al., 2006).

También hay que considerar al alumnado con NAE que participa activamente en la intimidación a otros compañeros (Kaukiainen et al., 2002). Se encuentra que acosan más los alumnos con problemas de conducta, con dificultades emocionales y de autocontrol (Carter y Spencer, 2006) y los que tienen conductas agresivas (Estell, Farmer, Irvin, Crowther, Akos y Boudah, 2009). Finalmente mencionar que pueden aparecer con un doble perfil de bully-víctima o como víctima-provocadora (Monchy, Pijl y Zandberg, 2004).

Teniendo en cuenta estos planteamientos, el presente estudio pretende analizar el rechazo y victimización social a los alumnos con NAE en comparación con sus compañeros sin NAE. Asimismo se pretende comprobar si existen diferencias entre los rechazados con y sin necesidad de apoyo educativo. Concretamente se plantean dos objetivos: 1) Analizar las diferencias en aceptación social, victimización, reputación social, competencia percibida y competencia social en alumnos con y sin necesidades de apoyo educativo y 2) Comprobar si existen diferencias entre alumnos rechazados con y sin necesidad de apoyo educativo en la reputación social entre los iguales y en la competencia social estimada por el profesorado.

De acuerdo con la evidencia de la investigación previa se formulan las siguientes hipótesis: Los alumnos con NAE comparados con sus compañeros sin NAE son más rechazados y más victimizados por sus iguales, tienen una reputación conductual de más agresivos, menos prosociales y más aislados, se autoperciben más negativamente y son valorados por sus profesores como menos competentes socialmente. Los alumnos rechazados y con NAE comparados con sus compañeros rechazados sin NAE: tienen similares puntuaciones en comportamiento prosocial, tienen puntuaciones más bajas en comportamientos antisociales y son valorados por sus profesores como menos competentes socialmente. 


\section{Método}

\section{Participantes}

Participaron un total de 1351 alumnos, 672 niños $(49.7 \%$ ) y 679 niñas $(50.3 \%)$, de 5 y 6 años de edad, de dos cohortes separadas por un año, escolarizados en 58 aulas de primer curso de educación primaria de 24 colegios públicos de áreas urbanas de Castellón (539 alumnos, 39.9\%), Palma de Mallorca (191 alumnos, 14.1\%), Sevilla (320 alumnos, $23.7 \%$ ) y Valladolid (301 alumnos, 22.3\%). La submuestra de los alumnos con necesidad de apoyo educativo (NAE) fue identificada por el profesorado según los criterios señalados en la introducción y está formada por 253 alumnos, lo que constituye el 18.7\% del alumnado. Son 168 chicos (66.4\%) y 85 chicas $(33.6 \%)$.

Concretamente, el profesorado ha considerado que 212 alumnos (el 83.8\%) de esta submuestra presenta necesidades de apoyo vinculadas a condiciones personales de deficiencias físicas, auditivas, visuales, discapacidad intelectual, dificultades de aprendizaje...), 28 alumnos (el 11.1\%) presentan problemas de conducta y 13 alumnos (el 5.1\%) tienen necesidades derivadas de especiales condiciones sociales, económicas, culturales, geográficas o étnicas. Hay que tener en cuenta que en determinados casos el niño presenta más de un problema, por ejemplo dificultades de aprendizaje y problemas de conducta y solamente se han adscrito a una categoría.

\section{Instrumentos}

Cuestionario de nominaciones sociométricas entre iguales.- Se utilizó una prueba sociométrica de nominación entre iguales con un criterio de preferencia directa (gusta estar), bidimensional positiva (compañeros o compañeras con los que te gusta estar más) y negativa (con aquellos que te gusta estar menos) y permitiendo nominaciones ilimitadas dentro del grupo clase. Los niños tenían que señalar, en una orla con las fotos de todos los alumnos de su clase, aquellos compañeros y compañeras con quienes le gusta estar más y con quienes le gusta estar menos (GREI, 2010). Este instrumento se pasó a todos los niños participantes. Los cálculos se han realizado con el programa Sociomet (González y García Bacete, 2010), que proporciona la identificación de los tipos sociométricos de cada aula. Los alumnos pueden ser identificados en uno de los cinco tipos sociométricos como preferidos, promedios, rechazados, ignorados o controvertidos.

Puntuación sociométrica de los iguales (Peer Rating).- Este procedimiento consiste en pedir a los participantes que estimen, generalmente según una escala de tipo Likert, el grado en que los otros compañeros del grupo cumplen un criterio, normalmente relacionado con gustar o jugar. Por ejemplo, ¿Te gustaría jugar con este niño? (1 = Nada o muy poco, $2=$ Algo, 3 = Bastante o mucho). Como señalan Asher y Dodge (1986), el método de calificaciones es similar a la clásica escala de dis- tancia social de Bogardus. En opinión de Cillessen (2009) está técnica, aunque no es tan popular como el método de nominaciones, se sigue usando frecuentemente. Frente a los métodos de nominación su principal desventaja es que requiere de mucho más tiempo por lo que a veces es prácticamente imposible obtener esta información en grupos grandes o en muestra grandes. Por el contrario, este método presenta una ventaja con respecto a los métodos de nominación, en tanto que asegura que todos los alumnos son valorados por todos sus compañeros, lo que puede aumentar su validez social. Los criterios utilizados han sido: (a) Me gusta (Liking): ¿Cuánto te gusta estar con........? (b) Prosocial-ayuda: ¿Cuánto ayuda a los demás?, (c) Timidez: ¿Es tímido/a? ¿Cuánta vergüenza le da estar con otros niños y niñas?, (d) Agresión-física: ¿Cuánto pega a los demás?, y (e) Olvidado: ¿Cuánto le buscan para jugar?; en este último, se invierte la puntuación para que mayor puntuación indique mayor olvido.

Competencia social estimada por el profesorado.- Consta de un solo ítem en el que se pidió al profesor-tutor de cada grupoaula que estimara la competencia social de cada alumno en una escala de 1 a 5 , desde muy incompetente a muy competente.

Juego de clase-revisado Revised Class Play, RCP, Wojslawowicz, Rubin, Burguess, Booth-LaForce y Rose-Krasnor, 2006, ampliado por GREI, 2010).- Se utilizó un formato clásico de nominación reputacional usando el Class Play (Masten, Morison y Pellegrino, 1985). El procedimiento consiste en pedir individualmente a los alumnos y alumnas que se imaginen los directores de una película o de una obra de teatro y que repartan una serie de papeles entre sus compañeros de clase teniendo en cuenta que sus características personales sean lo más parecidas posible a las del papel que deben representar. Para facilitar la comprensión de algunos ítems, se usaron unos dibujos con monigotes representando la situación descrita. Para cada una de las conductas evaluadas se calcula la proporción de veces que cada estudiante ha sido nombrado por el grupo para desempeñar ese papel.

Se partió de un amplio banco de ítems iniciales, tomados principalmente de la propuesta del Extended Class Play de Wojslawowicz et al., (2006), a la que se le añadieron ítems relacionados principalmente con agresión relacional (Crick, Casas y Mosher, 1997) y con popularidad percibida y dominancia social (Lease, Musgrove y Axelrod, 2002). Para el presente estudio sólo se utilizaron los ítems correspondientes a agresión, prosocialidad y timidez (25 ítems). Se realizaron pruebas exploratorias, análisis de ítems y análisis factoriales exploratorios (en adelante AFE) de componentes principales y rotación varimax. La prueba Kaiser-Meyer Olkin es .933 y la prueba de esfericidad de Barlett 11357.775 ( $g l=300$; $p<.000)$. Se han seleccionado los factores con autovalores mayores que 1. El AFE señala claramente una estructura factorial de tres factores: agresión, prosocialidad y aislamiento/timidez, que explican en torno a un $63 \%$ de la varianza (35.14\%, $18.43 \%$ y $9.01 \%$, respectivamente). Posteriormente 
se realizó un análisis factorial confirmatorio (en adelante AFC) mediante el método de ajuste de máxima probabilidad con estimaciones robustas (ML-Robusto, método). Para mostrar la adecuación del modelo se han utilizado los siguientes índices de ajuste: Satorra-Bentler Scaled $\chi 2$, S$\mathrm{B} \chi 2 / \mathrm{df}$, Bentler-Bonett Non-Normed Fit Index (BBNN), Comparative Fit Index (CFI) y Root Mean-Square Error of Aproximation (RMSEA). Los criterios para un buen ajuste son: índices próximos o inferiores a 2 en el índice $\mathrm{S}-\mathrm{B} \chi 2 / \mathrm{df}$, valores cercanos o superiores a .950 en los índices BBNN y CFI y valores inferiores a .05 en el índice RMSEA. Se consideran ajustes moderados o razonables cuando el índice $\mathrm{S}$ $\mathrm{B} \chi 2 / \mathrm{df}$ se sitúa entre 2 y 5 , los índices BBNN, CFI e IFI tienen valores iguales o superiores a $.90 \mathrm{y}$ los valores del valores el índice RMSEA son iguales o inferiores a .08. Los resultados obtenidos en el AFC por el método robusto confirman un modelo de tres factores, agresividad, prosocialidad y aislamiento $\left(\chi^{2} \mathrm{~s}-\mathrm{B}(98)=228.7616, p=0.000 ; \chi^{2} \mathrm{~s}-\mathrm{B} / \mathrm{df}=\right.$ 2.33 ; $\mathrm{BBNN}=0.933$; CFI $=.945$; RMSEA $=.046$, con el $90 \%$ del intervalo de confianza entre .038 y .053).

El factor de sociabilidad, formado por 5 ítems, incluía aspectos relacionados con la asertividad, las habilidades comunicativas y la conducta prosocial (sigue las reglas, ayuda a los demás). El factor de agresividad, formado por 6 ítems, incluía aspectos de agresión directa, agresión relacional e hiperactividad (se mete en peleas, dice a otro niño/ a: no puedes jugar con nosotros hasta que no hagas lo que te digamos, molesta a los demás cuando están trabajando). El factor de timidez/aislamiento, formado por 5 ítems, incluye aislamiento pasivo y timidez (es muy tímido/a, le da vergüenza, casi siempre está solo"). En cuanto al análisis de la fiabilidad, todos los factores, presentan buenos coeficientes alfa de Cronbach: prosocialidad, $\alpha=.90$; agresión $\alpha=.92$; timidez/aislamiento $\alpha=.72$.

Escala de victimización (GREI, 2010).- Se utilizó un autoinforme de 8 ítems construido ad hoc en el que se pide que el niño informe de la frecuencia (Nunca, Pocas veces, Bastantes veces, Casi todos los días) con la que, en el último mes, le han pasado diversas situaciones referidas a posible acoso y victimización por parte de sus compañeros. Son ejemplos: Algunos/as niños/as de la clase... te insultan, te ponen motes y te dicen cosas feas o desagradables, te dejan fuera de los juegos y no quieren estar contigo, se burlan y se ríen de ti. Para su validación, se realizó un AFE de componentes principales y rotación varimax. La prueba Kaiser-Meyer Olkin es .902 y la prueba de esfericidad de Barlett $2702.405(g l=28 ; p<.000)$. Se han seleccionado los factores con autovalores mayores que 1. Los 8 ítems forman un único factor (con un total de varianza explicada de $44.94 \%$ ). Finalmente se realizó un AFC por el método robusto, que indican que los 8 ítems informan de forma adecuada de un solo factor de victimización $\left(\chi^{2}\right.$ s-B $(20)=$ $26.2302, p=.158 ; \chi^{2} \mathrm{~s}$ - $/ \mathrm{df}=1.31 ; B B N N=.98 ; C F I=.99$; RMSE $A=.035$, con el $90 \%$ del intervalo de confianza entre .000 y .068), y con una alta fiabilidad $(\alpha=.82)$.
Escala gráfica de competencia percibida para niños (The Pictorial Scale of Perceived Competence and Social Acceptance for Young Children, Harter y Pike, 1983).- Este instrumento es una de las herramientas más citadas y usadas para evaluar la competencia percibida de los niños de cuatro a siete años. Consta de 24 ítems que evalúan las auto-percepciones de los niños en lo que respecta a la competencia física (por ejemplo, correr, saltar), la competencia cognitiva (por ejemplo, leer, contar), la aceptación entre los iguales (por ejemplo, tiene muchos amigos) y la aceptación materna (por ejemplo, mamá cocina la comida preferida de la niña). Cada ítem se presenta en una lámina con dos imágenes alusivas y una escala de 4 puntos. Se pide al niño/a que señale cuál es el niño/la niña más parecida a él/ella, ya que hay láminas con protagonistas niños y otras con niñas. En la presente investigación siempre que en el documento original se aludía a "mamá", hemos aludido a "mamá o papá".

Los resultados obtenidos en el $A F C$ por el método robusto confirman la estructura de 4 factores, cada uno con 6 ítems, propuesta por Harter y Pike (1983). Los índices informan de un buen ajuste de los datos al modelo $\left(\chi^{2} \mathrm{s-B}\right.$ (243) $=391.498, p=.000 ; \chi^{2} \mathrm{~s}-\mathrm{B} / \mathrm{df}=1.61 ; B B N N=.94 ; C F I=$ .95; RMSE $A=.027$, con el $90 \%$ del intervalo de confianza entre .022 y .031). El coeficiente de fiabilidad fue de .70 para el factor de percepción de competencia cognitiva, .54 para la percepción de competencia física, .77 para la aceptación de los pares percibido, y .68 para la aceptación percibida de los padres. Los índices de fiabilidad de la competencia percibida son aceptables, excepto el de percepción de la competencia física.

\section{Procedimiento}

Se contactó con diversos colegios públicos de Castellón, Palma de Mallorca, Sevilla y Valladolid y se les informó de los distintos pormenores de la investigación. Los centros contaron con el acuerdo del consejo escolar o del claustro de profesores del centro. Además se recabó la autorización firmada de la familia de los niños participantes.

La administración de los cuestionarios se inició a mediados del mes de noviembre de cada curso, una vez que el grupo-aula estaba estructurado después de dos meses de clases.

Teniendo en cuenta la edad de los participantes, 5 o 6 años, no-lectores o neolectores con una competencia lectora incipiente, todos los cuestionarios fueron administrados individualmente. Los niños fueron entrevistados por miembros del equipo investigador y por estudiantes entrenados para ello. Para agilizar al máximo el pase de cuestionarios, varios entrevistadores estaban simultáneamente en el mismo centro. Al finalizar cada sesión de evaluación se utilizaron estrategias distractoras; cada niño recibía una pequeña pegatina que se pegaba en la mano o una lámina para colorear, dibujar o recortar, a fin de que no hablara del tema al volver a clase y el contenido de las pruebas incidiera lo menos posible en la dinámica del aula. 
El análisis de los resultados se realizó con el paquete estadístico SPSS versión 19. Para el análisis de las diferencias, y comprobado que se cumplían los supuestos para el uso de pruebas paramétricas, se utilizó una prueba $t$ de Student para muestras independientes, calculando adicionalmente el tamaño del efecto $d$ de Cohen (1988), teniendo en cuenta la diferencia de tamaño muestral de los grupos, considerando un tamaño del efecto elevado, medio y bajo en función de los puntos de corte de $.80, .50$ y .20 respectivamente. En la comparación entre variables cualitativas, se han utilizado tablas de contingencia, calculando los residuos tipificados corregidos.

\section{Resultados}

En primer lugar, para la muestra total, se determinó la tipología sociométrica y se identificaron los rechazados. Los cálculos realizados con el programa Sociomet (González y García Bacete, 2010) dan como resultado los siguientes porcentajes de tipos sociométricos: Preferido (13.2\%), Rechazado $(13 \%)$, Ignorado $(3.9 \%)$, Controvertido $(1.6 \%)$ y Promedio $(68.2 \%)$. Estos resultados son coincidentes con otros estudios sobre la distribución sociométrica de las aulas, en donde se estima que alrededor del 15\% del alumnado es rechazado, y que, de ellos, aproximadamente el $75 \%$ son varones. En este caso, se identificaron un total de 176 alumnos rechazados, un 13\% del total. De ellos, 114 (64.8\%) eran varones y $62(35.2 \%)$ mujeres.

$\mathrm{Si}$ analizamos el porcentaje de alumnado con NAE dentro de cada tipo sociométrico (Tabla 1), vemos que, en general, son más frecuentes los niños con necesidad de apoyo dentro de los rechazados e ignorados, al contrario que en promedios y en especial en los preferidos, produciendo una distribución estadísticamente diferente en los grupos de promedios, preferidos y marcadamente entre los rechazados. En este sentido, mientras el $9.2 \%$ de los niños sin necesida- des son rechazados, aquellos con NAE forman el 29.2\% de este tipo sociométrico. Sin embargo, el $15.2 \%$ de los niños sin necesidades son preferidos, frente al $4.7 \%$ del alumnado con NAE. El porcentaje de promedios, aunque en menor medida, también es inferior en la submuestra de alumnado con NAE. En consecuencia, hay significativamente más probabilidad de que el alumnado con alguna Necesidad de Apoyo Educativo sea rechazado, y menos de ser promedio $y$, en especial, preferido.

Tabla 1. Alumnado con y $\sin$ NAE en cada tipo sociométrico.

\begin{tabular}{|c|c|c|c|c|c|}
\hline & & & & $\mathrm{AE}$ & \\
\hline & & & SÍ & $\mathrm{NO}$ & Total \\
\hline Tipo & Promedio & $f$ & $151^{* *}$ & $771 *$ & 922 \\
\hline sociométrico & & $\%$ & $59.7 \%$ & $70.3 \%$ & $68.3 \%$ \\
\hline & & RTC & -3.3 & 3.3 & \\
\hline & Preferido & $f$ & $12^{* *}$ & $167^{*}$ & 179 \\
\hline & & $\%$ & $4.7 \%$ & $15.2 \%$ & $13.3 \%$ \\
\hline & & $\mathrm{RTC}$ & -4.4 & 4.4 & \\
\hline & Rechazado & $f$ & $74^{*}$ & $101 * *$ & 175 \\
\hline & & $\%$ & $29.2 \%$ & $9.2 \%$ & $13 \%$ \\
\hline & & RTC & 8.5 & -8.5 & \\
\hline & Ignorado & f & 13 & 39 & 52 \\
\hline & & $\%$ & $5.1 \%$ & $3.6 \%$ & $3.9 \%$ \\
\hline & & RTC & 1.2 & -1.2 & \\
\hline & Controvertido & $f$ & 3 & 18 & 21 \\
\hline & & $\%$ & $1.2 \%$ & $1.6 \%$ & $1.6 \%$ \\
\hline & & $\mathrm{RTC}$ & -0.5 & 0.5 & \\
\hline
\end{tabular}

Por otra parte, como se puede ver en la Tabla 2, el alumnado con NAE informa de mayor maltrato que el alumnado que no tiene necesidades siendo estas diferencias significativas en el factor único de victimización y en aspectos como insultar, pegar, hacer llorar, hacer rabiar o excluir, aunque, en todos estos casos, el tamaño del efecto es bajo. No se aprecian diferencias significativas en los demás aspectos evaluados.

Tabla 2. Diferencias en victimización entre alumnado con y $\sin$ NAE.

\begin{tabular}{|c|c|c|c|c|c|c|c|}
\hline NAE & & $n$ & $M$ & $D T$ & $t$ & Sig. & $d$ \\
\hline \multirow[t]{2}{*}{ Algunos niños de la clase te insultan, te ponen motes.. } & SÍ & 233 & 2.13 & 1.16 & $2.950 * *$ & .003 & .23 \\
\hline & $\mathrm{NO}$ & 1037 & 1.88 & 1.05 & & & \\
\hline \multirow{2}{*}{...te empujan o te dan patadas } & SÍ & 233 & 2.23 & 1.12 & $3.277 * *$ & .001 & .25 \\
\hline & $\mathrm{NO}$ & 1038 & 1.97 & 1.03 & & & \\
\hline \multirow[t]{2}{*}{...te tratan mal o te hacen llorar } & SÍ & 233 & 2.02 & 1.12 & $3.809 * * *$ & .000 & .30 \\
\hline & $\mathrm{NO}$ & 1036 & 1.72 & 0.99 & & & \\
\hline \multirow[t]{2}{*}{...te pinchan, chinchan, hacen rabiar } & SÍ & 232 & 2.24 & 1.15 & $3.468 * *$ & .001 & .27 \\
\hline & $\mathrm{NO}$ & 1038 & 1.95 & 1.07 & & & \\
\hline \multirow[t]{2}{*}{...te dejan fuera de los juegos y no quieren estar contigo } & SÍ & 232 & 2.08 & 1.18 & $4.306 * * *$ & .000 & .35 \\
\hline & $\mathrm{NO}$ & 1036 & 1.72 & 0.99 & & & \\
\hline \multirow[t]{2}{*}{...te obligan a hacer cosas que no quieres hacer } & SÍ & 233 & 1.85 & 1.06 & 1.096 & .273 & .08 \\
\hline & $\mathrm{NO}$ & 1035 & 1.77 & 1.00 & & & \\
\hline \multirow[t]{2}{*}{...se burlan y se ríen de ti } & SÍ & 231 & 1.85 & 1.10 & 1.361 & .174 & .10 \\
\hline & $\mathrm{NO}$ & 1031 & 1.75 & 1.01 & & & \\
\hline \multirow[t]{2}{*}{... intentan que los otros niños no sean amigos míos } & SÍ & 231 & 1.81 & 1.07 & 1.444 & .150 & .11 \\
\hline & $\mathrm{NO}$ & 1038 & 1.70 & 0.99 & & & \\
\hline \multirow[t]{2}{*}{ Factor Único de Victimización } & SÍ & 228 & .50 & .18 & $3.946^{* * *}$ & .000 & .29 \\
\hline & $\mathrm{NO}$ & 1024 & .45 & .17 & & & \\
\hline
\end{tabular}

${ }^{*} p<.05 .{ }^{* *} p<.01 .{ }^{* * *} p<.001$ 
En cuanto a reputación social, tanto valorativa como identificativa a través de las pruebas de Puntuación sociométrica de los iguales (Peer Rating) y Juego de clase (Class Play) respectivamente (Tabla 3 ), se aprecia como el alumnado con NAE es significativamente menos valorado en Me gusta y en Ayuda, con un tamaño del efecto medio, que sus compañe- ros sin necesidades, mientras que son mucho más valorados, en orden creciente de significatividad, en timidez, agresividad y olvido, datos que se corroboran cuando se les pide que identifiquen aquellos roles para una obra de teatro o película en las dimensiones conductuales descritas.

Tabla 3. Diferencias en reputación social entre aquellos con y sin NAE.

\begin{tabular}{|c|c|c|c|c|c|c|c|c|}
\hline & NAE & & $n$ & $M$ & $D T$ & $t$ & Sig. & $d$ \\
\hline \multirow[t]{10}{*}{ Peer Rating } & \multirow[t]{2}{*}{ Me gusta } & SÍ & 185 & 1.89 & .45 & \multirow[t]{2}{*}{$-6.639 * * *$} & \multirow[t]{2}{*}{.000} & \multirow[t]{2}{*}{-.54} \\
\hline & & $\mathrm{NO}$ & 823 & 2.13 & .44 & & & \\
\hline & \multirow[t]{2}{*}{ Ayuda } & SÍ & 185 & 1.87 & .47 & \multirow[t]{2}{*}{$-9.492 * * *$} & \multirow[t]{2}{*}{.000} & \multirow[t]{2}{*}{-.76} \\
\hline & & $\mathrm{NO}$ & 823 & 2.23 & .47 & & & \\
\hline & \multirow[t]{2}{*}{ Timidez } & SÍ & 185 & 1.59 & .32 & \multirow{2}{*}{$4.097 * * *$} & \multirow[t]{2}{*}{.000} & \multirow[t]{2}{*}{.33} \\
\hline & & $\mathrm{NO}$ & 823 & 1.48 & .33 & & & \\
\hline & \multirow[t]{2}{*}{ Agresión } & SÍ & 185 & 1.62 & .63 & \multirow[t]{2}{*}{$5.367^{* * *}$} & \multirow[t]{2}{*}{.000} & \multirow[t]{2}{*}{.54} \\
\hline & & $\mathrm{NO}$ & 823 & 1.35 & .47 & & & \\
\hline & \multirow[t]{2}{*}{ Olvidado } & SÍ & 175 & 1.90 & .46 & \multirow[t]{2}{*}{$6.547^{* * *}$} & \multirow[t]{2}{*}{.000} & \multirow[t]{2}{*}{.59} \\
\hline & & $\mathrm{NO}$ & 757 & 1.65 & .41 & & & \\
\hline \multirow[t]{6}{*}{ Class Play } & \multirow[t]{2}{*}{ Agresividad } & SÍ & 253 & .13 & .14 & \multirow[t]{2}{*}{$5.495 * * *$} & \multirow[t]{2}{*}{.000} & \multirow[t]{2}{*}{.57} \\
\hline & & $\mathrm{NO}$ & 1096 & .07 & .09 & & & \\
\hline & \multirow[t]{2}{*}{ Prosocialidad } & SÍ & 253 & .05 & .04 & \multirow[t]{2}{*}{$-11.519 * * *$} & \multirow[t]{2}{*}{.000} & \multirow[t]{2}{*}{-.72} \\
\hline & & $\mathrm{NO}$ & 1096 & .10 & .07 & & & \\
\hline & \multirow[t]{2}{*}{ Aislamiento } & SÍ & 253 & .10 & .06 & \multirow[t]{2}{*}{$5.567 * * *$} & \multirow[t]{2}{*}{.000} & \multirow[t]{2}{*}{.54} \\
\hline & & $\mathrm{NO}$ & 1096 & .07 & .05 & & & \\
\hline
\end{tabular}

Respecto a la competencia percibida (ver Tabla 4), se encuentran, aunque en diferente grado, diferencias estadísticamente significativas en detrimento del alumnado con necesidad de apoyo, en competencia cognitiva percibida y aceptación de los iguales, con un tamaño del efecto medio y bajo respectivamente. Por el contrario no se aprecian diferencias entre los grupos en los otros dos factores evaluados, la percepción que tienen de la aceptación por parte de su familia y de la competencia física percibida.

Tabla 4. Diferencias en competencia percibida entre aquellos con y sin NAE.

\begin{tabular}{|c|c|c|c|c|}
\hline NAE & $M$ & $D T$ & $t$ & Sig. $d$ \\
\hline Aceptación de los & SÍ $215 \quad .82$ & .15 & $-2.605^{*}$ & $.010-.22$ \\
\hline iguales & NO1020.85 & .13 & & \\
\hline Competencia cogniti- & SÍ $\quad 217 \quad .82$ & .14 & $-4.650^{* * *}$ & $k^{k} .000-.44$ \\
\hline va percibida & NO1021.87 & .10 & & \\
\hline Aceptación de los & SÍ $217 \quad .69$ & .16 & .226 & .821 .00 \\
\hline padres & NO1016.69 & .15 & & \\
\hline Competencia física & SÍ $\quad 217 \quad .81$ & .13 & .667 & .505 .00 \\
\hline percibida & NO1017.81 & .12 & & \\
\hline
\end{tabular}
${ }^{*} p<.05 .{ }^{* *} p<.01 .{ }^{* * *} p<.001$

Por último, analizamos la percepción que tiene el profesorado sobre la competencia social de su alumnado, en donde nos encontramos con una menor puntuación para aquellos con NAE $(n=232, M=1.33, D T=1.64)$ frente a aquellos que no presentan necesidades $(n=1062, M=2.25, D T$ $=1.93)$, siendo esta diferencia altamente significativa $(p<$ $.001), t(1292)=-7.39, p<.000, d=-.49$, lo que evidencia que los alumnos con NAE son estimados, por parte del profesorado, como menos competentes que sus compañeros sin necesidad de apoyo.

Si profundizamos en los comportamientos concretos que diferencian a los alumnos que son rechazados y que tienen necesidad de apoyo de los rechazados que no tienen esas necesidades, podemos comprobar, como se muestra en la Tabla 5 , que aunque no aparecen diferencias significativas en ninguno de los tres factores analizados, hay una clara tendencia a recibir puntuaciones más altas, en las dimensiones reputacionales de agresividad y aislamiento, por parte del alumnado con NAE.

En la Tabla 6 se muestran los ítems que indican diferencias significativas entre estos dos subgrupos. El análisis pone de manifiesto que las diferencias van en detrimento de los alumnos rechazados y con NAE ya que corresponden a conductas relacionadas con comportamientos agresivos y disruptivos. No se han constatado diferencias significativas en los aspectos relativos a prosocialidad y sociabilidad.

Tabla 5. Diferencias en reputación social entre los rechazados con y sin NAE.

\begin{tabular}{|c|c|c|c|c|c|c|c|}
\hline$\overline{\mathrm{NAE}}$ & & $n$ & $M$ & $D T$ & $t$ & Sig. & $d$ \\
\hline \multirow[t]{2}{*}{ Agresividad } & SÍ & 74 & .25 & .17 & .950 & .053 & .30 \\
\hline & $\mathrm{NO}$ & 101 & .20 & .15 & & & \\
\hline \multirow[t]{2}{*}{ Prosocialidad } & SÍ & 74 & 03 & .02 & -1.229 & .221 & -.16 \\
\hline & $\mathrm{NO}$ & 101 & .04 & .03 & & & \\
\hline \multirow[t]{2}{*}{ Aislamiento } & SÍ & 74 & .14 & .07 & 1.960 & .052 & .30 \\
\hline & $\mathrm{NO}$ & 101 & .11 & .08 & & & \\
\hline
\end{tabular}

${ }^{*} p<.05 .{ }^{* *} p<.01 .{ }^{* * *} p<.001$ 
Tabla 6. Conductas en las que se producen diferencias entre rechazados con y sin NAE.

\begin{tabular}{|c|c|c|c|c|c|c|c|}
\hline$\overline{N A E}$ & & $n$ & $M$ & $D T$ & $t$ & Sig. & $d$ \\
\hline \multirow[t]{2}{*}{ Se mete en peleas } & SÍ & 74 & .33 & .27 & $2.286^{*}$ & .024 & .36 \\
\hline & $\mathrm{NO}$ & 101 & .24 & .23 & & & \\
\hline \multirow[t]{2}{*}{ Es muy tímido/a } & SÍ & 74 & .13 & .09 & $2.086^{*}$ & .038 & .32 \\
\hline & $\mathrm{NO}$ & 101 & .10 & .09 & & & \\
\hline \multirow[t]{2}{*}{ Le pegan, le dan patadas.... } & SÍ & 74 & .19 & .14 & $2.515^{*}$ & .013 & .41 \\
\hline & $\mathrm{NO}$ & 101 & .13 & .11 & & & \\
\hline \multirow[t]{2}{*}{... tiene buenas ideas para jugar } & SÍ & 74 & .04 & .05 & $-2.202^{*}$ & .029 & -.34 \\
\hline & $\mathrm{NO}$ & 101 & .06 & .05 & & & \\
\hline \multirow[t]{2}{*}{ Dice a otros niños: "Ya no eres mi amigo" } & SÍ & 74 & .18 & .14 & $2.129 *$ & .035 & .32 \\
\hline & $\mathrm{NO}$ & 101 & .14 & .12 & & & \\
\hline \multirow[t]{2}{*}{ No presta atención al profesor } & SÍ & 74 & .33 & .21 & $2.309 *$ & .022 & .35 \\
\hline & $\mathrm{NO}$ & 101 & .26 & .20 & & & \\
\hline \multirow[t]{2}{*}{ Es un/a llorica } & SÍ & 74 & .21 & .15 & $2.495^{*}$ & .014 & .38 \\
\hline & $\mathrm{NO}$ & 101 & .15 & .14 & & & \\
\hline \multirow[t]{2}{*}{ No gusta a los otros niños } & SÍ & 74 & .25 & .15 & $2.928 * *$ & .004 & .45 \\
\hline & $\mathrm{NO}$ & 101 & .18 & .14 & & & \\
\hline \multirow[t]{2}{*}{ Cuando no le salen las cosas, da pataletas, grita... } & SÍ & 74 & .27 & .21 & $3.610^{* * *}$ & .000 & .58 \\
\hline & NO & 101 & .16 & .15 & & & \\
\hline \multirow[t]{2}{*}{ Le eligen en último lugar } & SÍ & 74 & .19 & .11 & $2.245^{*}$ & .026 & .24 \\
\hline & $\mathrm{NO}$ & 101 & .16 & .11 & & & \\
\hline \multirow[t]{2}{*}{ Pega muchas veces } & SÍ & 74 & .33 & .27 & $2.082 *$ & .039 & .32 \\
\hline & $\mathrm{NO}$ & 101 & .24 & .23 & & & \\
\hline \multirow[t]{2}{*}{ Casi nunca está en su sitio } & SÍ & 74 & .31 & .23 & $3.069 * *$ & .003 & .48 \\
\hline & $\mathrm{NO}$ & 101 & .21 & .19 & & & \\
\hline Cuando no quiere escuchar a otro/a niño/a se da la vuel- & SÍ & 74 & .19 & .15 & $2.465^{*}$ & .015 & .39 \\
\hline ta... & $\mathrm{NO}$ & 101 & .14 & .11 & & & \\
\hline
\end{tabular}

${ }^{*} p<.05 .{ }^{* *} p<.01 .{ }^{* * *} p<.001$

Finalmente, respecto a la competencia social percibida por el docente, y dentro del grupo de rechazados, nos encontramos con una menor puntuación de aquellos con NAE $(n=71, M=1.07, D T=1.37)$ frente a aquellos que no presentan necesidades $(n=98, M=1.96, D T=1.63)$, siendo estas diferencias estadísticamente significativas $(p<.001), t$ $(167)=-3.83, p<.000$, y con un tamaño del efecto $d=-.57$.

\section{Discusión}

Este trabajo, que se ha focalizado en el alumnado que al inicio de la escolaridad obligatoria tiene necesidad de apoyo educativo, trata de esclarecer algunos aspectos referidos al rechazo y a la posible victimización que este grupo recibe de sus compañeros de clase. En síntesis, los resultados obtenidos evidencian que el alumnado con NAE, en comparación son sus compañeros del aula sin NAE, son más rechazados, tienen peor reputación social, se autoperciben más negativamente, se sienten víctimas de malos tratos y son considerados menos competentes por sus profesores. Es más, cuando se comparan alumnos rechazados con y sin NAE, los primeros tienen peor reputación social y el profesorado les califica más bajo en su competencia social.

Teniendo en cuenta la información aportada por todo el alumnado del aula, los resultados obtenidos indican que los alumnos con necesidades de apoyo educativo son más rechazados y tienen peor reputación social que sus iguales sin NAE.
Con respecto a las diferencias en aceptación social los datos de este estudio están en sintonía con el actual estado de conocimientos sobre este tema que resalta que este grupo es más rechazado y los porcentajes de rechazo encontrados en el alumnado con NAE son semejantes a los de autores como Frostad y Pijl (2007), aunque son menores que los señalados por otros estudios (Kavale y Forness, 1996; Vuran, 2005). Los resultados del presente trabajo son discrepantes con la tesis defendida por Frederickson (2010) y Frederickson y Furnham (2004) ya que en las aulas del presente estudio no se ha encontrado un trato benevolente del alumnado hacia sus compañeros con dificultades. Al contrario, reciben más nominaciones negativas hecho que pone de manifiesto que a sus compañeros no les gusta estar con ellos y prefieren la compañía de otros iguales sin NAE.

Considerando las diferencias en reputación social entre el alumnado con y sin NAE, este trabajo aporta resultados interesantes ya que ofrece un perfil reputacional del alumnado con necesidad de apoyo educativo de más agresión, más aislamiento/retraimiento y especialmente menos prosocialidad, ya que es el factor en el que se produce una diferencia más marcada entre ambos grupos. Estos aspectos coinciden exactamente con el patrón conductual característico del rechazo descrito repetidamente en la literatura. En efecto, como detallan autores como Bierman (2004) los niños rechazados son los menos sociables y prosociales, los más agresivos-disruptivos y los más aislados. Los resultados obtenidos al comprobar si existen diferencias en la competencia social estimada por el profesorado entre el alumnado con y sin 
NAE apoyan las predicciones hechas ya que el profesorado etiqueta como menos competente en el ámbito social al alumnado con NAE lo que está en sintonía con el corpus de conocimiento acumulado que informa de una visión negativa del rechazado por parte del profesorado (Nowicki. 2003; Pijl et al., 2010). Estos resultados son consistentes con la idea de que estos alumnos tienen dificultades sociales y carecen o son deficitarios en las habilidades sociales necesarias para relacionarse adecuadamente con los demás y para lograr su aceptación social y la integración activa en el grupo de pares y/o a la vez presentan conductas que resultan incómodas, aversivas y molestas (Estell et al., 2008). Aunque Frostad y Pijl (2007) sugieren que es una relación causa-efecto circular ya que si el niño no tiene habilidades sociales, no se relaciona con los otros y esa carencia de relaciones lleva a un insuficiente e inadecuado desarrollo de la competencia social, el presente estudio no permite concluir sobre las causas de los déficits sociales.

Por lo tanto, a la luz de estos datos y teniendo en cuenta la investigación sobre la relación entre la reputación social y el rechazo, se puede constatar la espiral del rechazo para el alumnado con NAE que viene a confirmar que el rechazo es un fenómeno grupal. Inicialmente el niño con NAE, por sus características o por sus déficits en habilidades sociales, pone en juego alguna conducta que molesta o irrita a los demás y el grupo reacciona con un "no me gusta" en forma de exclusión o rechazo explícito. Ante este rechazo el niño puede reaccionar de forma agresiva, disruptiva-molesta o replegándose y autoaislándose y el grupo intensifica las conductas de desagrado y exclusión. En este proceso el niño con NAE va adquiriendo una mala reputación entre sus pares, lo que va a acentuar las conductas de ignorancia y evitación de forma que el rechazo llega a ser un proceso grupal. El que el grupo de alumnado con NAE tenga esta imagen de agresivos, raros y con pocas habilidades sociales en su aula de pertenencia es un claro factor de riesgo de que se mantenga su estatus de rechazo y exclusión (García-Bacete et al., 2010; Gest, Rulison, Davidson, Welsh y Domitrovich, 2008).

En relación a la información que el propio alumnado ha aportado sobre sus percepciones y apreciaciones en las relaciones con los demás, los alumnos con NAE se autoperciben más negativamente que sus compañeros y no se sienten bien tratados por ellos.

Por lo que respecta al objetivo dedicado a conocer las diferencias en competencia percibida entre el alumnado con y sin NAE los resultados apoyan en parte lo esperado ya que el alumnado con NAE tiene peor autoconcepto que sus compañeros sin NAE en lo referente a competencia personal (competencia cognitiva percibida) y a la aceptación de los iguales; se sienten rechazados. Estos aspectos han sido previamente señalados por autores como Bakker y Bossman (2003), Cambra y Silvestre (2003) y Pilj y Frostad (2010) que informan de que tienen una percepción más negativa de su auto-imagen, de su competencia social, de su autoeficacia social y más baja autoestima y auto-confianza. No obstante en nuestro estudio no se aprecian diferencias significativas entre ambos grupos ni en la autopercepción que tienen de su propia competencia física ni en la de aceptación por parte de su familia. Sin embargo todas estas cuestiones han de ser consideradas con prudencia porque son escasos los estudios dedicados a analizar la autopercepción de competencia con esta población y en esta edad.

Si nos centramos en las diferencias en victimización entre el alumnado con y sin NAE los resultados obtenidos confirman la hipótesis planteada y son consistentes con los de investigaciones anteriores que afirman que esta población experimenta y siente exclusión, humillación y ostracismo por parte de sus iguales (Frederikson, 2010, y Frederikson et al., 2007, entre otros). Una cuestión que es preciso apuntar es que en este estudio se ha evaluado percepción de maltrato y sentimiento de víctima; no se ha evaluado el acoso en el sentido estricto de reiteración de las agresiones. Las preguntas se referían a la frecuencia de ocurrencia de determinados eventos en el último mes, periodo adecuado de recuerdo en niños de esta edad. Aunque las puntuaciones obtenidas en victimización no son muy altas el alumnado con NAE autoinforma, en mayor medida que sus compañeros sin NAE, de que recibe insultos, agresiones físicas, exclusión y aislamiento, situaciones que pueden llevar a acoso si se repiten sistemáticamente. Sin embargo, este subgrupo no percibe ese maltrato de sus iguales en otras circunstancias menos directas (te obligan a hacer cosas que no quieres; intentan que los otros niños no sean amigos míos). Hay que tener en cuenta que son niños de primero de educación primaria, y en consecuencia, con un grado de conciencia social todavía incipiente, lo que unido a las propias dificultades sociales mostradas (aislamiento, carencias de red de apoyo) hace que sean poco hábiles en reconocer situaciones de maltrato más indirectas y relacionales (Bourke y Bourgman, 2010; Rose et al., 2011).

La literatura científica permite explicar el proceso por el que los alumnos con NAE son el blanco de acoso, humillaciones y exclusiones sistemáticas. Generalmente el bullying se inicia con una conducta pro-activa agresiva (una broma, humillación, agresión, exclusión...) del intimidador que el niño-receptor no afronta o lo hace inadecuadamente. Esta respuesta estimula y provoca al acosador que repite la acción; si la víctima fracasa de nuevo, se inician los primeros eslabones de la cadena del acoso que se consolida a medida que el resto de los miembros del grupo van asumiendo distintos roles en la dinámica de intimidación-victimización (Monjas, 2009; Ortega y Mora, 2008; Sánchez et al., 2012). Aunque las investigaciones iniciales de Olweus (1998) apuntaban que las desviaciones externas negativas no inducían a la victimización, estudios posteriores han apoyado que diversas características físicas como la debilidad, los defectos o las diversas dificultades que presenta el alumnado con NAE, se asocian con mayor acoso. Así mismo se señala que un importante factor de riesgo es su baja competencia social y sus dificultades de integración y aceptación social (Frederikson, 2010; Rose et al., 2009; Whitney et al., 1992). El alumno con NAE es objetivo fácil para el acoso porque no tiene red de apoyo social en el grupo; no tiene amigos o tiene muy pocos 
y suelen ser otras víctimas u otros colegas también con dificultades que no pueden ofrecerles mucha ayuda contra la intimidación (Bollmer, Milich, Harris y Maras, 2005; Carter y Spencer, 2006; Perren y Alsaker, 2006; Thompson, Whitney y Smith, 1994).

En un intento de indagar y profundizar en los aspectos que diferencian a los rechazados con y sin NAE se formuló la hipótesis de que habría diferencias significativas en los perfiles reputacionales de ambos grupos en el sentido descrito por Frederikson (2010) de que los alumnos rechazados y con NAE tienen puntuaciones más bajas en comportamientos anti-sociales y tienen puntuaciones similares en comportamientos positivos. Los resultados obtenidos no apoyan totalmente esta hipótesis ya que los rechazados que también tienen NAE presentan en mayor medida comportamientos anti-sociales y disruptivos como son algunas conductas de inmadurez (cuando no le salen las cosas tiene rabietas, da pataletas, es un llorica...), disruptivas (no presta atención al profesor; no está en su sitio), agresivas (se mete en peleas; pega...). Sí se cumple que los perfiles reputacionales de ambos grupos son muy parecidos en el bajo funcionamiento positivo en aspectos como conductas prosociales (ayuda a los demás; defiende a los demás; comparte), afecto positivo (es amable; tiene sentido del bumor, es divertido...) y sociabilidad (tiene muchos amigos; soluciona pacificamente los conflictos...). Finalmente al comprobar si existen diferencias en la competencia social estimada por el profesorado entre alumnos rechazados con y sin NAE, los resultados han ratificado la hipótesis propuesta ya que el profesorado considera que, dentro de los alumnos rechazados, aquelos que tienen NAE tienen menor competencia social. Todo esto viene a indicar que los alumnos con NAE y que además son rechazados tienen una mayor problemática socioemocional y por tanto peor pronóstico que el alumnado sin NAE.

Como puede fácilmente deducirse de lo presentado, el sufrir rechazo o intimidación no puede considerarse exclusivamente una consecuencia directa de déficits y problemas individuales y personales de los participantes, sino que ambos fenómenos son psicosociales donde hay que considerar las respuestas de los iguales y las dinámicas sociales que ocurren en el seno del grupo. Como indican Juvonen y Gross (2004) estamos hablando de un conjunto de dificultades intrapersonales e interpersonales que conducen a y son resultado de procesos de rechazo y de acoso de los pares. Ser parte activa del grupo y afrontar asertivamente los intentos de acoso y violencia interpersonal son competencias muy complejas que requieren determinadas habilidades socioemocionales que los alumnos con NAE parecen no poseer. Desde luego si se quiere que el alumnado con NAE tenga interacciones positivas y amistad con sus compañeros, hemos de enseñarles cómo construir esas relaciones a través de programas de habilidades sociales y de otras estrategias trabajadas con el grupo de iguales (Estell, Jones, Peral y Van Acker, 2009; Flynt y Morton, 2004; Frederikson y Furnham, 2004; Meadan y Monda-Amaya, 2008; Monjas, 2008).
En resumen los principales hallazgos de este estudio reflejan los serios problemas de los alumnos con NAE que constituyen un colectivo en riesgo con una alta vulnerabilidad para experimentar procesos de rechazo y de acoso entre iguales. Este trabajo es el primer estudio realizado en nuestro país sobre esta temática, con esta población en esta edad y por tanto aporta resultados novedosos que contribuyen a ampliar el estado de conocimientos sobre rechazo y exclusión al alumnado con NAE.

Teniendo en cuenta el sufrimiento que ocasiona ser y sentirse rechazado y/o víctima de malos tratos por parte de los compañeros y las negativas consecuencias que esto conlleva para su desarrollo por la pérdida de oportunidades de aprendizaje y disfrute con los iguales, de este estudio se deducen diversas implicaciones prácticas. La primera tiene que ver con la necesidad de formación al profesorado para hacerles conscientes tanto de los problemas específicos de esta población en estas temáticas como de su influencia en las dinámicas del aula y en la creación de contextos y redes de apoyo para el alumnado con dificultades y también de la conveniencia de enfatizar los aspectos socio-emocionales y promover una educación positiva en el sentido apuntado por Seligman, Ernst, Gillham, Reivich y Linkins (2009) como la educación tanto para las habilidades tradicionales como para la felicidad. La segunda apela a la importancia del trabajo con los iguales del grupo que pueden ser un importante factor de resiliencia para reducir la vulnerabilidad de los potenciales compañeros rechazados o víctimas (Bierman, 2004; Bollmer et al., 2005; Gallagher, Dadisman, Farmer, Huss y Hutchins, 2007; Margalit, 2004; Von Grünigen, Perren, Nägele y Alsaker, 2010). La tercera implicación es que la comprensión más precisa de este fenómeno, puede posibilitar el diseño de intervenciones más eficaces con este grupo. A este respecto se propone la intervención universal con el grupo-clase a través de, entre otros, programas socioemocionales, metodología cooperativa, erradicación de sesgos reputacionales, potenciación de la amistad y establecimiento de redes de ayuda entre iguales, y también la intervención más específica con los alumnos con NAE y con los rechazados. Remarcamos la conveniencia de que estas actuaciones se hagan en el marco de los planes de mejora de la convivencia y de promoción de relaciones entre iguales de calidad, integrando la prevención y la intervención dentro del currículo y de las rutinas escolares y, por supuesto, con el protagonismo del profesorado y los profesionales de apoyo (García Bacete et al., 2013).

Este trabajo tiene varias limitaciones como son la edad de los participantes y el poco tiempo que llevaban juntos en el aula, lo que ha implicado un largo y complejo proceso de recogida de datos; la gran diversidad y heterogeneidad de características de la submuestra del alumnado con NAE que, como hemos señalado previamente, presentan dificultades en muy diversos aspectos y la escasa literatura científica en nuestro país sobre esta temática y población que ha obligado a tomar como referencia investigaciones desarrolladas en otros países, con sistemas educativos difícilmente compara- 
bles con el nuestro. Por eso los hallazgos que se presentan han de tomarse con prudencia y, en algunos casos, más que resultados definitivos han de considerarse líneas de investigación futura. Pese a estas limitaciones, creemos que este trabajo puede orientar futuras investigaciones en las que se profundice en las relaciones aquí analizadas, contribuyendo de este modo, a mejorar la comprensión del problema. En este sentido es preciso estimular más investigación en nuestro país con los alumnos con NAE, ampliando la franja de edad y profundizando en las diversas necesidades y discapacidades. Sería conveniente hacer estudios longitudinales para analizar qué ocurre con ellos a lo largo de la escolaridad, evaluar sus fortalezas y aspectos positivos, las redes sociales y los grupos de compañeros con los que se juntan, evaluar el acoso teniendo en cuenta la reiteración de las conductas de

\section{Referencias}

Al-Yagon, M. y Mikulincer, M. (2004). Patterns of close relationships and socioemotional and academic adjustment among school-age children with learning disabilities. Learning Disabilities Research and Practice, 19(1), 12-19.

Asher, S. R. y Dodge, K. A. (1986). Identifying children who are rejected by their peers. Developmental Psychology, 22, 444-449.

Avilés, J. M. y Monjas, M. I. (2008). Estudio de incidencia de la intimidación y el maltrato entre iguales en la educación secundaria obligatoria mediante el Cuestionario CIMEI (Avilés, 1999), Cuestionario sobre intimidación y maltrato entre iguales. Anales de Psicologia, 21(1), 27-41.

Avramidis, E. (2010). Social relationships of pupils with special educational needs in the mainstream primary class: peer group membership and peer-assessed social behaviour. European Journal of Special Needs Education, 25(4), 413-429.

Bakker, J. T. A. y Bosman, A. M. T. (2003). Self-image and peer acceptance of dutch students in regular and special education. Learning Disability Quarterly, 26(1), 5-14.

Bakker, J. T. A., Denessen, E., Bosman, A. M. T., Krijger, E. y Bouts, L. (2007). Sociometric status and self-image of children with specific and general learning disabilities in dutch general and special education classes. Learning Disability Quarterly, 30(1), 47-62.

Baydik, B. y Bakkaloglu, H. (2009). Predictors of sociometric status for low socioeconomic status elementary mainstreamed students with and without special needs. Kuram Ve Uygulamada Egitim Bilimleri, 9(2), 435445.

Bierman, K. L. (2004). Peer rejection. Developmental, processes and intervention strategies. New York, NY: The Guilford Press.

Bollmer, J., Milich, R., Harris, M. y Maras, M. (2005). A friend is need: the role of friendship quality as a protective factor in peer victimization and bullying. Journal of Interpersonal Violence, 20(6), 701-12.

Bourke, S. y Bourgman, I. (2010). Coping with bullying in Australian schools: how children with disabilities experience support from friends, parents and teachers. Disability and Society, 25(3), 359-371.

Buelga, S., Cava, M. y Musitu, G. (2012). Reputación social, ajuste psicosocial y victimización entre adolescentes en el contexto escolar. Anales de Psicología, 28(1), 180-187.

Cambra, C. y Silvestre, N. (2003). Students with special educational needs in the inclusive classroom: social integration and self-concept. European Journal of Special Needs Education, 18(2), 197-208.

Carter, B. B. y Spencer, V. G. (2006). The fear factor: Bullying and students with disabilities. International Journal of Special Education, 21(1), 11-23.

Cerezo, F. (2009). La violencia en las aulas. Análisis y propuestas de intervención. Madrid: Pirámide.

Cerezo, F. y Ato, M. (2010). Estatus social, género, clima del aula y bullying entre estudiantes adolescentes. Anales de Psicología, 26(1), 137-144. maltrato, analizar la participación de alumnado con NAE en el rol de acosadores y profundizar en la evaluación de sus auto-percepciones. Sería interesante asimismo estudiar con detalle al pequeño porcentaje de alumnos con NAE que son resilientes y no experimentan rechazo ni victimización.

Agradecimientos.- Investigación realizada gracias a la ayuda de investigación "Rechazo entre iguales y dinámica social en el aula: una aproximación multidisciplinar y multimetodológica" (PSI200800541) concedida por el Ministerio de Ciencia e Innovación (Plan Nacional de I+D+I. 2008-2011).

Queremos hacer explícito nuestro agradecimiento a los centros educativos participantes y a los estudiantes universitarios colaboradores.

Cillessen, A. H. N. (2009). Sociometric Methods. En K. H. Rubin, W. Bukowski y B. Laursen (Eds.), Handbook of peer interactions, relationships, and groups (pp. 82-99). New York, NY: The Guilford Press.

Cillessen, A. H. N., Bukowski, W. M. y Haselager, G. J. T. (2000). Stability of sociometric categories. En A. H. N. Cillessen y W. M. Bukowski (Eds.), Recent advances in the measurement of acceptance and rejection in the peer system (pp. 75-93). San Francisco, CA: Jossey-Bass Inc.

Cohen, J. (1988). Statistical Power Analysis for the Behavioral Sciences. Hillsdale, New Jersey, NJ: Lawrence Erlbaum Associates.

Coie, J. D. (1990). Toward a theory of peer rejection. En S. R. Asher y J. D. Coie (Eds.), Peer rejection in childhood (pp 365-401). Cambridge, England: Cambridge University Press.

Crick, N. R., Casas, J. F. y Mosher, M. (1997). Relational and overt aggression in preschool. Developmental Psychology, 33(4), 579-587.

Dixon, R., Smith, P. y Jenks, C. (2004). Bullying and difference: a case study of peer group dynamics in one school. Journal of School Violence, 3(4), 4158.

Eguren, P., Gutiérrez, H., Herrero, M. I. y López, J. L. (2006). Maltrato y discapacidad visual. Psicología Educativa, 12 (1), 1135-755.

Escobar, M., Fernández Baena, F., Miranda, J., Trianes, M. V. y Cowie, H. (2011). Baja aceptación de los iguales e inadaptación emocional/conductual en escolares: Efectos del estrés cotidiano, afrontamiento y sexo. Anales de Psicología, 27(2), 412-417.

Estell, D. B., Farmer, T. W., Irvin, M. J., Crowther, A., Akos, P. y Boudah, D. J. (2009). Students with exceptionalities and the peer group context of bullying and victimization in late elementary school. Journal of Child and Family Studies, 18(2), 136-150.

Estell, D. B., Jones, M. H., Pearl, R. y Van Acker, R. (2009). Best friendships of students with and without learning disabilities across late elementary school. Exceptional Children, 76(1), 110-124.

Estell, D. B., Jones, M. H., Pearl, R., Van Acker, R., Farmer, T. W. y Rodkin, P. C. (2008). Peer groups, popularity, and social preference trajectories of social functioning among students with and without learning disabilities. Journal of Learning Disabilities, 41(1), 5-14.

Evans, D. Healey, E. C., Kawai, N. y Rowland, S. (2008). Middle school students' perceptions of a peer who stutters. Journal of Fluency Disorders, 33(3), 203-219.

Farmer, T. W. (2000). Misconceptions of peer rejection and problem behavior - understanding aggression in students with mills disabilities. Remedial and Special Education, 21(4), 194-208.

Flynt, S. W. y Morton, R. C. (2004). Bullying and children with disabilites. Journal of Instructional Psychology, 31(4), 330-333.

Frederickson, N. (2010). Bullying or befriending? Children's responses to classmates with special needs. British Journal of Special Education, 37(1), 412

Frederickson, N. y Furnham, A. F. (2004). Peer-assessed behavioural characteristics and sociometric rejection: Differences between pupils who 
have moderate learning difficulties and their mainstreaming peers. British Journal of Educational Psychology, 74, 391-410.

Frederikson, N., Simmonds, E., Evans, L. y Soulsby, C. (2007). Assessing the social and affective outcomes of inclusion. British Journal of Special Education, 34(2), 105-115.

Frostad, P. y Pijl, S. J. (2007). Does being friendly help in making friends? The relation between the social position and social skills of pupils with special needs in mainstream education. European Journal of Special Needs Education, 22(1), 15-30.

Gallagher, K. C., Dadisman, K. Farmer, Th. W., Huss, L. y Hutchins, B. C. (2007). Social dynamics of early childhood classrooms: Considerations and implications for teachers, En O. Saracho y B. Spodek (Eds.), Contemporary Perspectives in Early Childhood Education: Vol. 7. Social Learning in Early Childhood Education (pp. 17-48). Greenwich, CT: Information Age Publishing.

Garaigordobil, M. y Oñederra, J. A. (2010). Inteligencia emocional en las víctimas de acoso escolar y en los agresores. European Journal of Education and Psychology, 3(2), 243-256

García Bacete, F. J., Jiménez, I., Muñoz, M. V., Monjas, M. I., Sureda, I., Ferrá, P., Martín-Antón, L. J., Marande, G. y Sanchíz, M. L. (2013). Aulas como contextos de aceptación y apoyo para integrar a los alumnos rechazados. Apuntes de Psicología, 31(2), 11-20.

García Bacete, F. J., Sureda, I. y Monjas, M. I. (2008). Distribución sociométrica en las aulas de chicos y chicas a lo largo de la escolaridad. Revista de Psicología Social, 23(1), 63-74.

García Bacete, F. J. Sureda, I. y Monjas, M. I. (2010). El rechazo entre iguales en la educación primaria: una panorámica general. Anales de Psicología, 26(1), 123-136.

Gest, S. D., Rulison, K. L., Davidson, A. J., Welsh, J. A. y Domitrovich, C. E. (2008). A reputation for success (or failure): The association of peer academic reputations with academic self-concept, effort, and performance across the upper elementary grades. Developmental Psychology, 44(3), 625-636.

Gifford-Smith, M. E. y Brownell, C. A. (2003). Childhood peer relationships: Social acceptance, friendships and peer networks. Journal of School Psychology, 41, 235-284

González, J. y García Bacete, F. J. (2010). Sociomet. Programa para la realización de estudios sociométricos. Madrid: TEA Ediciones.

Granizo, L., Na, P. y del Barrio, C. (2008). Análisis de las relaciones sociales de los alumnos con Síndrome de Asperger en escuelas integradas de secundaria: un estudio de casos. Revista de Psicodidáctica, 11(2), 281-292.

GREI (2010). Rechazo entre iguales y dinámica social en el aula: una aproximación multidisciplinar y multimetodológica (Memoria del Proyecto PSI2008004541). Castellón: Universidad Jaume I de Castelló.

Harris, M. J. (2009). Bullying, rejection, and peer victimization: A social cognitive neuroscience perspective. New York, NY: Springer.

Harter, S. y Pike, R. (1983). The Pictorial Scale of Perceived Competence and Social Acceptance for Young Children. Denver, CO: University of Denver.

Heinrichs, R. R. (2003). A whole school approach to bullying: Special considerations for children with exceptionalities. Intervention in School and Clinic, 38(4), 195-204.

Hernández, J. M. y van der Meulen, K. (2010). El maltrato por abuso de poder en el alumnado con trastornos del espectro autista y sus efectos sobre la inclusión. Siglo Cero, 41 (1), 23-40.

Hugh-Jones, S. y Smith, P. K. (1999). Self-report of short and long term effects of bullying of children who stammer. British Journal of Educational Psychology, 69 (2), 141-158.

Jiang, X. L. y Cillessen, A. H. N. (2005). Stability of continous measures of sociometric status: a meta-analysis. Developmental Review, 25, 1-25.

Juvonen, J. y Grahan, S. (2001) (Eds.). Peer harassment in school. New York, NY: Guilford Press.

Juvonen, J. y Gross, E. F. (2004). The Social Outcast: Ostracism, Social Exclusion, Rejection, and Bullying. En Williams, K. D., Forgas, J. P. y von Hippel, W. (Eds.), The social outcast: Ostracism, social exclusion, rejection, and bullying. New York, NY: Psychology Press.

Kaukiainen, A., Salmivallii, C., Lagerspetz, K., Vauras, M., Maki, H. y Poskiparta, E. (2002). Learning difficulties, social intelligence and selfconcept: connections to bully-victim problems. Scandinavian Journal of Psychology, 43(3), 269-278.
Kavale, K. y Forness, S. (1996). Social skill deficits and learning disabilities: A meta-analysis. Journal of Learning Disabilities, 29(3), 226-237.

Kemp, C. y Carter, M. (2002). The social skills and social status of mainstreamed students with intellectual disabilities. Educational Psychology, 22(4), 391-411.

Koster, M., Pijl, S. J., van Houten, E. y Nakken, H. (2007). The social position and development of pupils with SEN in mainstream Dutch primary schools. European Journal of Special Needs Education, 22(1), 31-46.

Lease, A. M., Musgrove, K. T. y Axelrod, J. L. (2002). Dimensions of social status in preadolescent peer groups: likability, perceived popularity, and social dominance. Social Development. 11(4), 508-533.

Lindsay, G., Dockrell, J. E. y Mackie, C. (2008). Vulnerability to bullying in children with a history of specific speech and language difficulties. European Journal of Special Needs Education, 23(1), 1-16.

Lindsay, S. y McPherson, A. M. (2012). Experiences of social exclusion and bullying al school among children and youth with cerebral palsy. Disability and Rehabilitation, 34(2), 101-109.

Lucas, B., Pulido, R. y Solbes, I. (2011). Violencia entre iguales en Educación Primaria: el papel de los compañeros y su relación con el estatus sociométrico. Psicothema, 23(2), 245-251.

Mand, J. (2007). Social position of special needs pupils in the classroom: comparison between German special schools for pupils with learning difficulties and integrated primary school classes. European Journal of Special Needs Education, 22(1), 7-14.

Margalit, M. (2004). Second-generation research on resilience: Socialemotional aspects of children with learning disabilities. Learning Disabilities Research and Practice, 19(1), 45-48.

Masten, A. S., Morison, P. y Pellegrini, D. S. (1985). A revised class play method of peer assessment. Developmental Psychology, 21(3), 523-533.

Meadan, H. y Monda-Amaya, L. E., (2008). Collaboration to promote social competence for students with mild disabilities in the general classroom: A structure for providing social support. Intervention in School and Clinic, 43, 158-167.

Monchy, M. D., Pijl, S. J. y Zandberg, T. (2004). Discrepancies in judging social inclusion and bullying of people with behaviour problems. European Journal of Special Needs Education, 19(3), 317-330.

Monjas, M. I. (Dir.) (2008). Cómo promover la convivencia: Programa de asertividad $y$ habilidades sociales (PAHS). Madrid: CEPE. ( $1^{\mathrm{a}} \mathrm{ed} ., 3^{\mathrm{a}}$ imp.).

Monjas, M. I. y Avilés, J. M. (2006). Programa de sensibilización contra el maltrato entre iguales. Valladolid: Junta de Castilla y León y REA (Asociación castellano-leonesa para la defensa de la infancia y la juventud). ( $2^{\mathrm{a}}$ ed. revisada).

Monjas, M. I. (2009). Acoso entre iguales. El maltrato que sufren, ejercen y observan los menores entre ellos. En Castanyer, O. (Coord.), La víctima no es culpable. (pp. 111-148). Bilbao: DDB.

Nabuzoka, D. y Smith, P. K. (1993). Sociometric status and social behaviour of children with and without learning difficulties. Journal of Child Psychology and Psychiatry, 34(8), 1435-1448.

Nakamoto, J. y Schwartz, D. (2010). Is peer victimization associated with academic achievement? A meta-analytic review. Social Development, 19(2), 221-242.

Nakken, H. y Pijl, S. J. (2002) Getting along with classmates in regular schools: a review of the effects of integration on the development of social relationships. International Journal of Inclusive Education, 6(1), 47-61.

Norwich, B. y Kelly N. (2004). Pupils' views on inclusion: moderate learning difficulties and bullying in mainstream and special schools. British Educational Research Journal, 30(1), 43-65.

Nowicki, E. A. (2003). A meta-analysis of the social competence of children with learning disabilities compared to classmates of low and average to high achievement. Learning Disability Quaterly, 26(3), 171-088.

Olweus, D. (1998). Conductas de acoso y amenaza entre escolares. Madrid: Morata.

ORDEN EDU/1152/2010, de 3 de agosto, por la que se regula la respuesta educativa al alumnado con necesidad específica de apoyo educativo escolarizado en el segundo ciclo de Educación Infantil, Educación Primaria, Educación Secundaria Obligatoria, Bachillerato y Enseñanzas de Educación Especial, en los centros docentes de la Comunidad de Castilla y León (BOCYL de 13 de agosto).

Ortega, R. y Mora, J. (2008). Las redes de iguales y el fenómeno del acoso escolar: explorando el esquema dominio-sumisión. Infancia y Aprendizaje, 31(4) 515-528. 
Perren, S. y Alsaker, F. D. (2006). Social behavior and peer relationships of victims, bully-victims and bullies in kindergarten. Journal of Child Psychology and Psychiatry, and Allied Disciplines, 47(1), 45-57.

Pijl, S. J. (2007). Introduction: the social position of pupils with special needs in regular education. European Journal of Special Needs Education, 22(1), 1-5.

Pijl, S. J. y Frostad, P. (2010). Peer acceptance and self-concept of students with disabilities in regular education. European Journal of Special Needs Education, 25(1), 93-105.

Piil, S. J., Frostad, P. y Flem, A. (2010). The social position of pupils with special needs in regular schools. Scandinavian Journal of Educational Research, 52(4), 387-405.

Rose, C. A., Espelage, D. L. y Monda-Amaya, L. E. (2009). Bullying and victimization rates among students in general and special education: A comparative analysis. Educational Psychology, 29(7), 761-776.

Rose, C. A., Monda-Amaya, L. E. y Espelage, D. (2011). Bullying perpetration and victimization in special education: A review of the literature. Remedial and Special Education, 32(2), 114-130.

Ruijs, N. M. y Peetsma, T. D. (2009). Effects of inclusion on students with and without special educational needs reviewed. Educational Research Review, 4, 67-79.

Sabeh, E. y Monjas, M. I. (2002). La aceptación social de niños y niñas con discapacidad. Problemas y posibles soluciones. Siglo Cero, 33(2), 15-26.

Sánchez, V., Ortega, R. y Menesini, E. (2012). La competencia emocional de agresores y víctimas de "bullying". Anales de Psicología, 28(1), 71-82.

Savage, R. (2005). Friendship and bullying patterns in children attending a language base in a mainstream school. Educational Psychology in Practice, 21(1), 23-36.

Scheepstra, A. J. M., Nakken, H. y Pijl, S. J. (1999). Contacts with classmates: the social position of pupils with Down's syndrome in Dutch mainstream education. European Journal of Special Needs Education, 14(3),
212-220

Seligman, M. E. P., Ernst, R. M., Gillham, J., Reivich, K. y Linkins, M. (2009). Positive education: Positive psychology and classroom interventions. Oxford Review of Education, 35(3), 293.

Symes, W. y Humphrey, N. (2010). Peer-group indicators of social inclusion among pupils with autistic spectrum disorders (ASD) in mainstream secondary schools: A comparative study. School Psychology International, 31(5), 478-494.

Thompson, D., Whitney, I. y Smith, P. K. (1994). Bullying of children with special needs in mainstream schools. Support for Learning, 9(3), 103-106.

Vaugham, S., Elbaum, B. E. y Schumm, J. S. (1996). The effects of the inclusion on the social functioning of students with learning disabilities. Journal of Learning Disabilities, 29, 598-608.

Von Grunigen, R., Perren, S., Nägele, C. y Alsaker, F. D. (2010). Inmigrant children's peer acceptance and victimization in kindergarten: The rol of local language competence. The British Psychological Society, 88, 679-697.

Vuran, S. (2005). The sociometric status of students with disabilities in elementary level integration classes in Turkey. Eurasian Journal of Educational Research, 18, 236-252.

Warden, D. y MacKinnon, S. (2003). Prosocial children, bullies and victims: An investigation of their sociometric status, empathy and social problem-solving strategies. British Journal of Developmental Psychology, September (367-385).

Whitney, I., Nabuzoka, D. y Smith, P. K. (1992). Bullying in schools: Mainstream and special needs. Support for Learning, 7(1), 3-7.

Wojslawowicz, J. C., Rubin, K. H., Bowker, J. C., Booth-LaForce, C., Burgess, K. B. y Rose-Krasnor, L. (2006). Behavioral characteristics associated with stable and fluid best friendship patterns in middle childhood. Merrill-Palmer Quarterly, 52(4), 671-693.

(Articulo recibido: 16-9-2012; revisado: 27-3-2013; aceptado: 21-5-2013) 